\title{
Few-cycle optical solitary waves in nonlinear dispersive media
}

\author{
Sh. Amiranashvili and U. Bandelow \\ Weierstrass Institute for Applied Analysis and Stochastics, Mohrenstraße 39, 10117 Berlin, Germany \\ N. Akhmediev \\ Optical Sciences Group, Research School of Physics and Engineering, Institute of Advanced Studies, \\ The Australian National University, Canberra ACT 0200, Australia \\ (Received 19 July 2012; revised manuscript received 5 November 2012; published 4 January 2013)
}

\begin{abstract}
We study the propagation of few-cycle optical solitons in nonlinear media with an anomalous, but otherwise arbitrary, dispersion and a cubic nonlinearity. Our approach does not derive from the slowly varying envelope approximation. The optical field is derived directly from Maxwell's equations under the assumption that generation of the third harmonic is a nonresonant process or at least cannot destroy the pulse prior to inevitable linear damping. The solitary wave solutions are obtained numerically up to nearly single-cycle duration using the spectral renormalization method originally developed for the envelope solitons. The theory explicitly distinguishes contributions between the essential physical effects such as higher-order dispersion, self-steepening, and backscattering, as well as quantifies their influence on ultrashort optical solitons.
\end{abstract}

DOI: 10.1103/PhysRevA.87.013805

PACS number(s): 42.81.Dp, 42.65.Tg, 05.45.Yv, 42.65.Re

\section{INTRODUCTION}

Optical solitons are robust localized pulses arising from the interplay of nonlinearity and dispersion of the media. They are usually described by the pulse envelope governed by the nonlinear Schrödingier equation (NLSE) [1,2]. The latter is known to be integrable [3] with its fundamental soliton solution being the focus of studies in nonlinear optics. As the NLSE is derived using the slowly varying envelope approximation (SVEA), such a fundamental soliton must contain a sufficiently large number of optical oscillations. Recent progress in generation of few-cycle [4,5] and even subcycle [6-8] optical pulses boosted interest to ultrashort optical solitons. Several exact solutions have been found both for the generalized NLSE beyond the SVEA [9-14] and for the nonenvelope propagation equations derived specifically to describe ultrashort pulses [15-21]. When considering these solutions and in the present work, it is assumed that the pulse carrier frequency $\omega_{0}$ is well separated from any resonant frequency of the medium. Another important case of the resonant ultrashort solitons [22-24] is beyond the scope of our work.

Nonenvelope solitons are not based on SVEA and seem to be less universal than the fundamental soliton of the NLSE. The exact solutions known so far require either specific relations between the parameters of the generalized NLSE or simplified dispersion relations. For example, Drude's dispersion model in a wide spectral window is required for derivation of the so-called short pulse equation $[25,26]$.

Typically nonenvelope solitons belong to continuous families and they are characterized by the pulse duration $t_{0}$, among other parameters. As the pulse duration increases, the ultrashort soliton moving along the $z$ axis with velocity $V$ approaches the standard $\cosh ^{-1}\left(\tau / t_{0}\right)$ shape for $\tau=t-z / V$ and the fundamental soliton of the NLSE is recovered for $\omega_{0} t_{0} \gg 1$. This simple limit motivates a backward search for the few-cycle solitons. Let us first start with a fundamental soliton of the NLSE and transform it into an exact solution of the properly simplified set of Maxwell equations. By doing so we avoid the use of the envelope approximation and the NLSE. Thereafter one can trace the solution shape while solving Maxwell's equations and decreasing the soliton duration. This turns out to be an effective strategy at least for systems where nonlinear generation of the third harmonics can be ignored even for spectrally wide short pulses.

We have found that envelope solitons can be naturally transformed into ultrashort solitary solutions. These solutions can be traced down to nearly single-cycle duration but only as long as one can ignore contributions of both the higher harmonic generation and the soliton-specific Cherenkov radiation $[27,28]$. If this is the case, one can even observe formation of a nonsmooth cusp profile which prohibits existence of subcycle solitons. This fact agrees with the properties of the exact ultrashort solitary solutions obtained previously both for the generalized NLSE [14] and for the nonenvelope propagation model with idealized dispersion $[19,20]$. From the practical side, the ultrashort solitons presented in this paper provide natural initial conditions for direct modeling of solitons using Maxwell's equations, where imperfect ultrashort fundamental solitons are used instead [29-31]. The nonenvelope ultrashort solitons are obtained numerically using a simple and effective procedure, the so-called spectral renormalization method, originally developed for the NLSE [32].

\section{BASIC EQUATIONS}

For the sake of simplicity, we consider a single-mode waveguide and characterize the propagating pulse by a single field component. The latter depends on the propagation distance and time, $E=E(z, t)$, as prescribed by the scalar nonlinear wave equation

$$
\partial_{z}^{2} E-\frac{1}{c^{2}} \partial_{t}^{2}\left(\hat{\epsilon} E+\chi^{(3)} E^{3}\right)=0,
$$

where $c$ is the speed of light and the parameter $\chi^{(3)}$ is the nonlinear susceptibility of third order. For simplicity we take $\chi^{(3)}=$ const, although most of our results can be formulated for any $\chi^{(3)}\left(\omega_{1}+\omega_{2}+\omega_{3}, \omega_{1}, \omega_{2}, \omega_{3}\right)$. The full-scale 
dispersion of $\chi^{(3)}$ is commonly unknown for the frequency domains we are interested in. Therefore a phenomenological approach with instant cubic nonlinearity is more advantageous in general formulation. On the other hand, the peak intensity of the shortest one-cycle solitary solution remains moderate, $\chi^{(3)} E^{2} \ll 1$, and the higher-order nonlinearities can be ignored.

The nonlocal pseudodifferential operator $\hat{\epsilon}$ is defined by a suitable medium response function $h(t)$,

$$
\hat{\epsilon} E(z, t)=E(z, t)+\int_{0}^{\infty} E\left(z, t-t^{\prime}\right) h\left(t^{\prime}\right) d t^{\prime},
$$

such that for a single harmonic $E \sim e^{-i \omega t}$ we obtain

$$
(\hat{\epsilon} E)_{\omega}=\epsilon(\omega) E_{\omega}, \quad \epsilon(\omega)=1+\int_{0}^{\infty} h\left(t^{\prime}\right) e^{i \omega t^{\prime}} d t^{\prime},
$$

where $E_{\omega}=E_{\omega}(z)$ denotes the Fourier component of $E(z, t)$. The dielectric constant $\epsilon(\omega)$ accounts for the combined medium and waveguide dispersion.

Note that any nontrivial $\epsilon(\omega)$ from Eq. (2) is a complexvalued function. The corresponding dispersion relation

$$
\beta^{2}(\omega) c^{2}=\omega^{2} \epsilon(\omega)
$$

applies to a monochromatic linear wave with $E(z, t)$ proportional to exp $i(\beta z-\omega t)$. Equation (3) yields a complex-valued propagation constant $\beta(\omega)$ for each frequency. In other words, damping is in the very nature of Eq. (1), and all optical solitons are a priori approximate solutions in the transparency window. Consequently, looking for a soliton, one has a right to ignore, e.g., higher harmonics and Cherenkov radiation, if their effect is below the linear damping. In practice, such a soliton is a long-living robust solution that persists despite other pulses being destroyed by nonlinearity and dispersion, but it still can be destroyed by damping.

Optical pulses which propagate with a constant velocity without changing their shape, $E=E(t-z / V)$, were examined in Ref. [33]. This study is based on Eq. (1) with a realvalued positive, but otherwise arbitrary, dielectric constant. The solutions may only be expected if $\operatorname{Re}[\epsilon(\omega)]$ has either a maximum at some $\omega$ for a focusing nonlinearity or a minimum for a defocusing one. Unfortunately, this is not the case for the vast majority of material dispersions in the transparency window, i.e., all extrema of $\operatorname{Re}[\epsilon(\omega)]$ typically correlate to a high damping. The lack of the ultrashort solitons is in sharp contrast to the variety of the envelope solitons. The latter exist for any carrier frequency $\omega=\omega_{0}$ in the transparency window provided that $\chi^{(3)}$ and $\beta_{2}=\beta^{\prime \prime}\left(\omega_{0}\right)$ are of different sign. To be specific, we assume focusing nonlinearity and anomalous dispersion:

$$
\chi^{(3)}>0 \text { and } \beta^{\prime \prime}(\omega)<0 .
$$

The latter condition applies to some interval of frequencies around $\omega_{0}$. Normal dispersion is allowed in the transparency window outside this region.

In what follows, we show how to obtain nonenvelope solitary wave solutions for Eq. (1). To this end, one has to ignore contributions of higher harmonics. The most natural way to do so is to use the analytic signal representation for the electric field [34]. This approach is also a useful tool for the treatment of the pulse propagation problem such as the one presented in [35].

\section{REDUCTION}

Keeping in mind that we are interested in describing solitons for arbitrary dispersion, at some stage we have to deal with the numerical solution. It is then convenient to replace a continuous $\omega$ by a sufficiently dense discrete set of frequencies by introducing a large period $T$ in the time domain. Thus, the electric field is represented by a discrete sum

$$
E(z, t)=\sum_{\omega} E_{\omega}(z) e^{-i \omega t}, \quad \omega \in \frac{2 \pi}{T} \mathbb{Z},
$$

and

$$
E_{\omega}(z)=\int_{-T / 2}^{T / 2} E(z, t) e^{i \omega t} \frac{d t}{T}, \quad E_{-\omega}=E_{\omega}^{*} .
$$

Note that $E(z, t)$ and $E_{\omega}(z)$ have the same physical dimension. Performing numerical calculations, we actually keep only part of the harmonics, namely, those belonging to the transparency window

$$
\omega_{L}<|\omega|<\omega_{R},
$$

where $\omega_{L}$ and $\omega_{R}$ are suitable frequencies in the infrared and ultraviolet regions. Of course, all our results can be easily rewritten to apply to a continuous spectrum.

In what follows, we assume that the four-wave resonance conditions

$$
\begin{aligned}
\beta\left(\omega_{1}\right)+\beta\left(\omega_{2}\right)+\beta\left(\omega_{3}\right) & =\beta\left(\omega_{4}\right), \\
\omega_{1}+\omega_{2}+\omega_{3} & =\omega_{4}, \quad \omega_{i}>0,
\end{aligned}
$$

are not satisfied simultaneously for any four positive frequencies from the transparency window (7), whether they are discrete or not. Consequently, generation of the third harmonics is a nonresonant process.

To utilize the above feature, we introduce a complex field $\mathcal{E}(z, t)$ which by definition is governed by the equation

$$
\partial_{z}^{2} \mathcal{E}-\frac{1}{c^{2}} \partial_{t}^{2}\left(\hat{\epsilon} \mathcal{E}+\frac{3}{4} \chi^{(3)}|\mathcal{E}|^{2} \mathcal{E}+\frac{1}{4} \chi^{(3)} \mathcal{E}^{3}\right)=0
$$

where $\hat{\epsilon} \mathcal{E}$ is naturally defined in the frequency domain

$$
\hat{\epsilon} \mathcal{E}(z, t)=\sum_{\omega} \epsilon(\omega) \mathcal{E}_{\omega}(z) e^{-i \omega t},
$$

and $\mathcal{E}_{\omega}(z)$ denotes Fourier components of $\mathcal{E}(z, t)$. We stress that $\mathcal{E}$ is a complex-valued field and therefore $\mathcal{E}_{-\omega}$ and $\left(\mathcal{E}_{\omega}\right)^{*}$ are different, in contrast to the standard relation (6). Actually the positive-frequency part of $\mathcal{E}(z, t)$ will dominate over the negative-frequency part (see below).

In what follows, $\mathcal{E}_{\omega}^{*}$ always denotes a complex conjugate of $\mathcal{E}_{\omega}$. Fourier components of the conjugated field $\mathcal{E}^{*}$ are denoted by $\left(\mathcal{E}^{*}\right)_{\omega}$. One can check that

$$
\mathcal{E}_{\omega}^{*}=\left(\mathcal{E}_{\omega}\right)^{*}=\left(\mathcal{E}^{*}\right)_{-\omega},
$$

cf. Eq. (6). In addition, using (10), (11), and the standard relation $\epsilon(-\omega)=\epsilon^{*}(\omega)$ one can directly check that

$$
(\hat{\epsilon} \mathcal{E})^{*}=\hat{\epsilon}\left(\mathcal{E}^{*}\right)
$$


which extends the standard fact that $\hat{\epsilon}$ transforms an arbitrary real field into a real one.

Equation (9) is just an equivalent way to solve Eq. (1). Indeed, adding Eq. (9) to its complex conjugate, using Eq. (12), and defining

$$
E(z, t)=\frac{\mathcal{E}(z, t)+\mathcal{E}^{*}(z, t)}{2},
$$

we immediately recover Eq. (1) for the real electric field. To solve Eq. (1) one may introduce the complex field such that initially $E=\operatorname{Re}[\mathcal{E}$ ], then solve Eq. (9), and then recover $E(z, t)$ from (13).

Now we take advantage of the presumably nonresonant dispersion profile by choosing a special initial value for $\mathcal{E}(z, t)$. As usual, we deal with the waves propagating along the $z$ axis so that the initial state of the system is that for $z=0$. We present the incoming field $E(z=0, t)$ as a sum of harmonics (5) and define $\mathcal{E}(z=0, t)$ as the positive-frequency part of this sum,

$$
E(0, t) \rightarrow \mathcal{E}(0, t)=2 \sum_{\omega>0} E_{\omega}(0) e^{-i \omega t}
$$

where the factor 2 is for $E=\operatorname{Re}[\mathcal{E}]$. The time-averaged field $\langle E\rangle=E_{\omega=0}$ is set to zero, and the initial value of the derivative $\partial_{z} \mathcal{E}$ is related to $\partial_{z} E$ in a manner similar to Eq. (14).

Initially $\mathcal{E}(z=0, t)$ contains only positive frequencies and thus is an analytic signal for $E(z=0, t)$. Strictly speaking, this is not true for $z>0$, because negative-frequency components of $\mathcal{E}(z, t)$ still may be generated by the $|\mathcal{E}|^{2} \mathcal{E}$ term. However, the process is nonresonant. For example, let us assume that a harmonic $e^{-i \omega t}$ results from the nonlinear interaction of three positive-frequency harmonics $e^{-i \omega_{i} t}$ with $i=1,2,3$ through the $|\mathcal{E}|^{2} \mathcal{E}$ term, such that

$$
\begin{aligned}
\beta(\omega) & =\beta\left(\omega_{1}\right)-\beta\left(\omega_{2}\right)+\beta\left(\omega_{3}\right), \\
\omega & =\omega_{1}-\omega_{2}+\omega_{3}, \quad \omega_{i}>0 .
\end{aligned}
$$

If $\omega<0$, the latter conditions are equivalent to Eq. (8) and the resonance excitation does not occur. Therefore, to a large extent, $\mathcal{E}(z, t)$ contains only positive frequencies also for $z>0$.

Up to this very moment the transformation from Eq. (9) to Eq. (1) was exact. Now we take advantage of the above considerations and neglect generation of the third harmonics by ignoring the $\mathcal{E}^{3}$ term in Eq. (9). The resulting propagation equation

$$
\partial_{z}^{2} \mathcal{E}-\frac{1}{c^{2}} \partial_{t}^{2}\left(\hat{\epsilon} \mathcal{E}+\frac{3}{4} \chi^{(3)}|\mathcal{E}|^{2} \mathcal{E}\right)=0
$$

is the one that will be used below. All meaningful solutions of Eq. (15) are subject to a simple criterion: the negativefrequency part of $\mathcal{E}(z, t)$, being zero at $z=0$ by construction, must remain small for $z>0$.

Note that we keep the resonant term $|\mathcal{E}|^{2} \mathcal{E}$ and ignore the nonresonant one $\mathcal{E}^{3}$, even though both terms have the same order of magnitude. The reason is that the resonant effects are accumulated in propagation, whereas the nonresonant contributions are significantly smaller. In other words, the condition (8) ensures that higher harmonics, while observable, do not have sufficient feedback to influence the main part of the spectrum. Moreover, the nonresonant nonlinearities
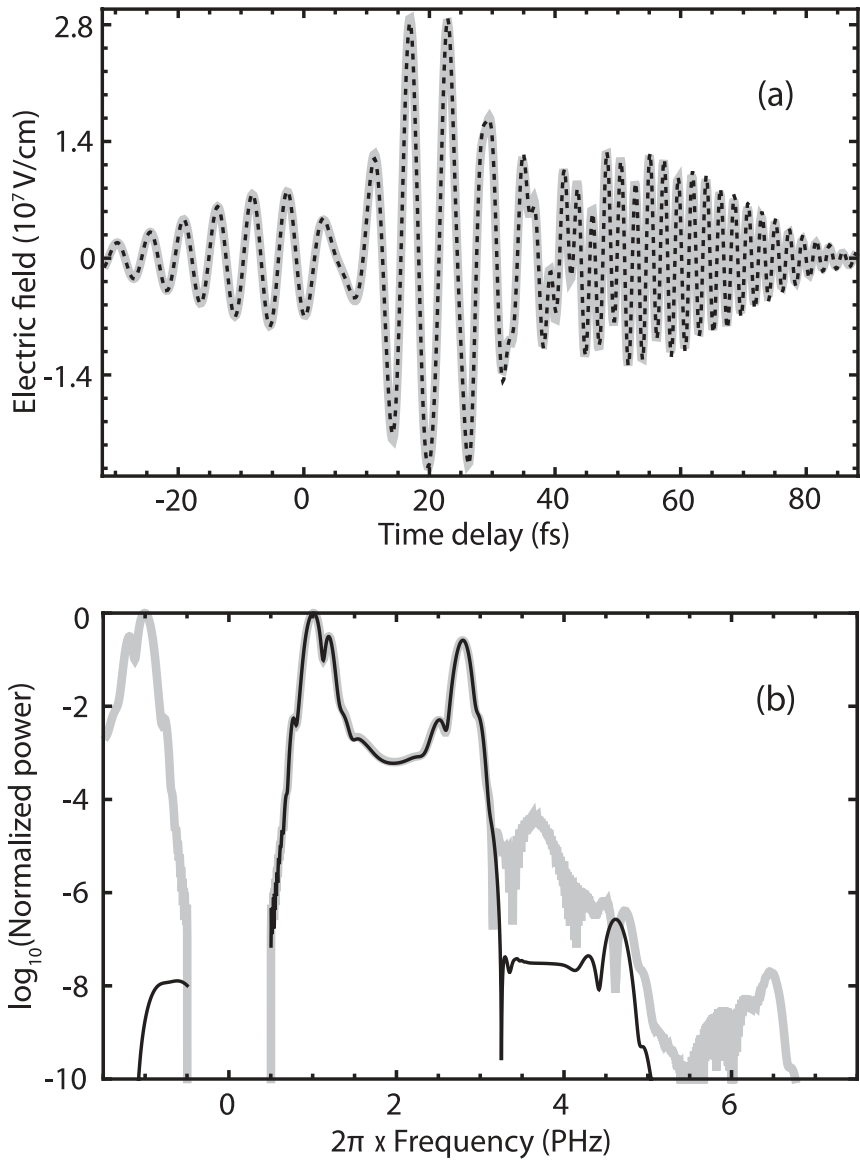

FIG. 1. (a) Electric field of a pulse at $z=3000 \mu \mathrm{m}$ which initially had three-cycle $1 /$ cosh shape. (b) Spectral density of the same pulse. The two time and spectral series are obtained solving the full Eq. (1) (gray curves) and the reduced Eq. (15) (black curves). Simulations are done for a pulse propagating in bulk fused silica at $\lambda=1.6 \mu \mathrm{m}$. The full set of parameters is given in the text.

can to some extent be eliminated by a suitable change of variables [36].

We illustrate the role of the resonant and the nonresonant terms by the direct comparison of numerical solutions of the reduced Eq. (15) with those of the full Eq. (1). Specifically, we consider a pulse propagating in bulk fused silica which initially had a shape of $\cosh ^{-1}\left(\tau / t_{0}\right)$. The carrier wavelength $\lambda=1.6 \mu \mathrm{m}$. We choose the initial pulse duration $2 t_{0}=16 \mathrm{fs}$ that corresponds to three optical cycles. The dispersion length $t_{0}^{2} /\left|\beta_{2}\right| \approx 1860 \mu \mathrm{m}$, while the nonlinear length $\approx 465 \mu \mathrm{m}$. We choose the initial amplitude to be twice that of the fundamental soliton. Then the corresponding peak power is $\approx 2 \times 10^{12} \mathrm{~W} / \mathrm{cm}^{2}$.

Figure 1 shows the electric field and the power spectrum of this pulse after propagation distance $z=3000 \mu \mathrm{m}$. The pulse changes its shape as the initial condition is chosen to be different from the stationary pulse. Importantly, in this complicated dynamics, the full and the reduced models result in practically identical real-valued fields, as can be seen from Fig. 1(a). Moreover, Fig. 1(b) shows that the main parts of the spectra of the two pulses are also identical. Deviations observed at high positive frequencies are 4 orders of magnitude smaller and they do not influence the main part of the 
spectrum. The parts of the spectra at negative frequencies $\omega<0$ also appear as expected: $\left|E_{\omega}\right|$ is symmetric, whereas $\left|\mathcal{E}_{\omega}\right|$ is asymmetric and 8 orders of magnitude smaller, i.e., can be completely ignored. The fact that the negative-frequency part of $\mathcal{E}(z, t)$ is negligible in pulse propagation dynamics is a crucial validity test of the reduced Eq. (15).

We conclude that Eq. (15) is an accurate replacement of the full Eq. (1) for investigations of the solitary wave solutions. For what follows, it is convenient to write Eq. (15) in the frequency representation

$$
\partial_{z}^{2} \mathcal{E}_{\omega}+\beta^{2}(\omega) \mathcal{E}_{\omega}+\frac{3 \chi^{(3)} \omega^{2}}{4 c^{2}}\left(|\mathcal{E}|^{2} \mathcal{E}\right)_{\omega}=0,
$$

where $\left(|\mathcal{E}|^{2} \mathcal{E}\right)_{\omega}$ denotes a spectral component of $|\mathcal{E}|^{2} \mathcal{E}$.

To summarize this section we stress that Eq. (15) has the same nonlinearity as the NLSE. It has a rich set of short-pulse solutions which are close to fundamental solitons. On the other hand, Eq. (15) is more general than the NLSE as it accounts for arbitrary dispersion and is not the result of the SVEA. An important generalization is that Eq. (15) takes into account waves moving in both directions, in contrast to all unidirectional envelope equations. Another key feature of Eq. (15) is that it keeps the initially zero or negligible negativefrequency part of $\mathcal{E}(z, t)$ to be negligible in propagation. Any resonant excitation of the higher-order harmonics, if it happens by, e.g., shock formation, immediately results in a quick growth of the negative-frequency components. Clearly, when it occurs, one should use the full Eq. (1). Last but not least, Eq. (15) is independent of the carrier frequency. Figure 1, for instance, suggests that the initial pulse is split in two subpulses with the considerably different frequencies. Still both subpulses are described by a single Eq. (15).

\section{SOLITONS}

We now turn to the investigation of the solitary waves. The latter move in one direction but nevertheless appear to be affected by the presence of the backward wave. Up until now the dielectric constant was an arbitrary complex-valued function for which $\operatorname{Re}[\epsilon(\omega)] \gg \operatorname{Im}[\epsilon(\omega)]$ in the transparency window (7). From now on we neglect the imaginary part completely and simply write $\epsilon(\omega)$ instead of $\operatorname{Re}[\epsilon(\omega)]$. All derived values, e.g., the propagation constant $\beta(\omega)$ and the refraction index $n(\omega)$, are then real.

Looking for the solitary wave solutions of Eq. (15), we do not have to assume $\mathcal{E}=\mathcal{E}(t-z / V)$ like in the case of Eq. (1), which required the ansatz $E=E(t-z / V)$ used previously in [33]. The propagation model given by Eq. (15) is more flexible than Eq. (1). To describe the solitary waves we introduce a complex-valued shape function $f(\tau)$ :

$$
f(\tau)=\sum_{\Omega} f_{\Omega} e^{-i \Omega \tau}, \quad \tau=t-\frac{z}{V},
$$

where $\tau$ refers to the retarded time and the parameter $V$ is the velocity of the soliton. We further introduce the carrier frequency $\omega_{0}$, defined such that

$$
\frac{1}{V}=\beta^{\prime}\left(\omega_{0}\right)
$$

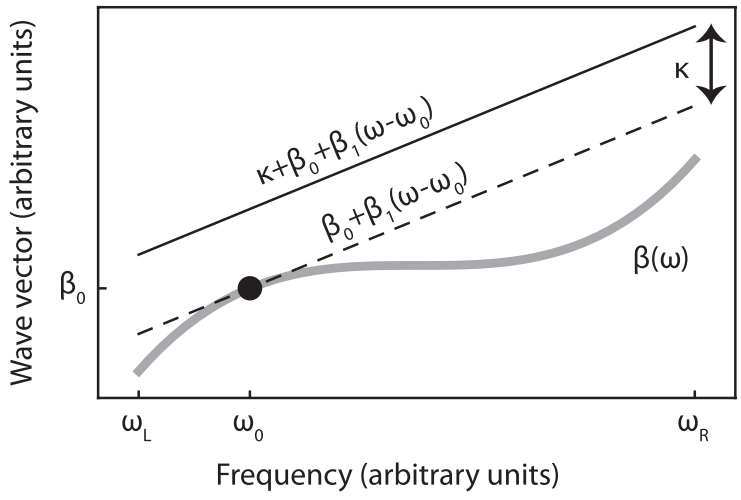

FIG. 2. A schematic representation of the dispersion relation $\beta=\beta(\omega)$ in the transparency window $\omega_{L}<\omega<\omega_{R}$. Solitons with the carrier frequency $\omega_{0}$ are parametrized by $\kappa$. They can be found numerically for sufficiently small $\kappa$ if $\omega_{0}$ belongs to the anomalous dispersion region and if the tangent line at $\omega_{0}$ does not intersect with $\beta(\omega)$. The fundamental soliton of the NLSE is recovered for $\kappa \rightarrow 0$.

and use the standard notation

$$
\beta_{m}=\beta^{(m)}\left(\omega_{0}\right)
$$

for the derivatives of $\beta(\omega)$ at $\omega=\omega_{0}$. We take advantage of another standard notation [37],

$$
n_{2}=\frac{3 \chi^{(3)}}{8 n\left(\omega_{0}\right)}
$$

and use $\sqrt{n_{2}}$ to normalize fields if necessary.

The soliton field is assumed to be of the form

$$
\mathcal{E}(z, t)=f\left(t-\beta_{1} z\right) e^{i\left(\kappa+\beta_{0}\right) z-i \omega_{0} t},
$$

where $\kappa$ is a correction to the propagation constant $\beta_{0}$ that results from the nonlinear terms in Eq. (15). The yet-unknown shape function $f(\tau)$ is parametrized by $\kappa$ and should converge to the fundamental soliton for $\kappa \rightarrow 0$. Note that a possible correction to $\omega_{0}$ can be "included" in $f(\tau)$ without loss of generality. In what follows, we use a notation

$$
\tilde{\beta}(\Omega)=\beta\left(\omega_{0}+\Omega\right)-\beta_{0}-\beta_{1} \Omega=\sum_{m=2}^{\infty} \frac{\beta_{m}}{m !} \Omega^{m}
$$

to characterize how $\beta(\omega)$ deviates from the tangent line at $\omega=\omega_{0}$ (see Fig. 2). Note that the above Taylor expansion, as well as any Taylor-expansion-based generalized NLSE, is valid only within the convergence radius. A simple way to improve convergence using no more than the standard set of propagation constants $\beta_{m}$ is discussed in [38].

The ansatz (18) in the frequency domain becomes

$$
\mathcal{E}_{\omega_{0}+\Omega}(z)=f_{\Omega} e^{i\left(\kappa+\beta_{0}+\beta_{1} \Omega\right) z} .
$$

In addition, for the $|\mathcal{E}|^{2} \mathcal{E}$ term, we have

$$
\left(|\mathcal{E}|^{2} \mathcal{E}\right)_{\omega_{0}+\Omega}=\left(|f|^{2} f\right)_{\Omega} e^{i\left(\kappa+\beta_{0}+\beta_{1} \Omega\right) z} .
$$

Inserting Eq. (19) into Eq. (16), we can write the result in the frequency domain in the form

$$
[\kappa-\tilde{\beta}(\Omega)] f_{\Omega}=\frac{\omega_{0}+\Omega}{c} \frac{n\left(\omega_{0}\right) n_{2}}{n\left(\omega_{0}+\Omega\right)+\eta}\left(|f|^{2} f\right)_{\Omega},
$$


where, for brevity, we used the notation

$$
\eta=\frac{\kappa-\tilde{\beta}(\Omega)}{\omega_{0}+\Omega} \frac{c}{2} .
$$

Equation (20) describes the solitary wave solutions we are interested in. For convenience it is written in terms of the carrier frequency $\omega_{0}$ and the deviation $\Omega=\omega-\omega_{0}$. However, Eq. (20) is not based on the SVEA. Actually, $\omega_{0}$ is just a reference frequency defined by Eq. (17). Before proceeding further, it is instructive to examine relations between Eq. (20) and different versions of the NLSE. A more detailed comparison is given in the Appendix and here we briefly outline the results.

Fundamental solitons of the classical NLSE [1,2] appear if one neglects dispersion in the right-hand side of Eq. (20) and replaces $\tilde{\beta}(\Omega)$ with its leading term for $\Omega \ll \omega_{0}$ in the left-hand side (SVEA),

$$
\left(\kappa-\frac{\beta_{2}}{2} \Omega^{2}\right) f_{\Omega}=\frac{\omega_{0}}{c} n_{2}\left(|f|^{2} f\right)_{\Omega} .
$$

We recall that $\beta_{2}<0$ and $n_{2}>0$ in accord with Eq. (4). Each $\kappa>0$ sets the fundamental soliton solution

$$
\sqrt{n_{2}} f(\tau)=\sqrt{\frac{2 \kappa c}{\omega_{0}}} \frac{1}{\cosh \left[\tau / t_{0}(\kappa)\right]},
$$

where the parameter

$$
t_{0}(\kappa)=\sqrt{\frac{\left|\beta_{2}\right|}{2 \kappa}}
$$

determines the duration of the soliton. Note, that $1 / \kappa$ defines, apart from a factor of order 1, the dispersion length.

Solitary wave solutions of the commonly used generalized NLSE [39] equipped by the full dispersion operator and the self-steepening term on the right-hand side are described by

$$
[\kappa-\tilde{\beta}(\Omega)] f_{\Omega}=\frac{\omega_{0}+\Omega}{c} n_{2}\left(|f|^{2} f\right)_{\Omega} .
$$

This equation is the same as Eq. (20), if one neglects $\eta$ and assumes that $n\left(\omega_{0}+\Omega\right) \approx n\left(\omega_{0}\right)$ for the pulse widths of interest.

Nonlocal NLSE has been introduced previously by several authors $[38,40-43]$. One spatial dimensional solitons in this approach are described by the relation

$$
[\kappa-\tilde{\beta}(\Omega)] f_{\Omega}=\frac{\omega_{0}+\Omega}{c} \frac{n\left(\omega_{0}\right) n_{2}}{n\left(\omega_{0}+\Omega\right)}\left(|f|^{2} f\right)_{\Omega},
$$

where the term $n\left(\omega_{0}+\Omega\right)$ in denominator is responsible for effective dispersion of $n_{2}$. This term contributes to nonlinear dispersion; the latter is then present even when $\chi^{(3)}=$ const. In addition, the full-scale dispersions of $\chi^{(3)}$ can be taken into account, but this will result in more cumbersome expressions.

Comparing Eq. (25) with Eq. (20), we can notice that only the $\eta$ term is missing in Eq. (25). This $\eta$ term is responsible for bidirectional propagation modeled by Eq. (15). Clearly, it disappears in the unidirectional models.

Let us now return to the most general Eq. (20). It takes into account the higher-order linear dispersion, self-steepening, effective nonlinear dispersion, and the parallel wave propagating backward. An equation mathematically similar to
Eq. (20) has been studied previously in [33]. Thus similar analysis applies also to our case. A distinguishing feature, when compared to Eq. (22), is that the factor $[\kappa-\tilde{\beta}(\Omega)]$ is not always positively defined. Moreover, the factor may vanish for some frequency (or set of frequencies) $\Omega_{r}$. When $\kappa \rightarrow 0$, i.e., for the fundamental soliton, the corresponding resonant frequency $\omega_{r}=\omega_{0}+\Omega_{r}$ is determined by the equation

$$
\omega_{r}-\beta\left(\omega_{r}\right) V=\omega_{0}-\beta\left(\omega_{0}\right) V .
$$

We recall here that by definition $V=\left[\beta^{\prime}\left(\omega_{0}\right)\right]^{-1}$ is the soliton velocity. Equation (26) simply indicates that Doppler-shifted frequencies of the resonant wave and the carrier wave are identical in the comoving frame. In such cases there are no localized solutions of Eq. (20). In practice, if $\omega_{r}$ belongs to the spectrum of the pulse, the latter continuously emits the so-called Cherenkov radiation [27,28] and thus cannot be a soliton in the standard definition. On the other hand, the resonant radiation is exponentially small if $\omega_{r}$ is well separated from $\omega_{0}$. This, in turn, requires a large region with the anomalous dispersion where $\beta(\omega)$ is a convex function (see Fig. 2). Transforming Eq. (20) into the time domain, we obtain a robust localized solution which in reality is slowly deformed by the combined effects of radiation, dissipation, and the third harmonics generation.

If the resonant radiation can be ignored, each carrier frequency in the anomalous dispersion domain generates a family of solitary wave solutions parametrized by $\kappa>0$. The fundamental soliton solution (23) of the NLSE derived in the SVEA is recovered from Eq. (20) when $\kappa \rightarrow 0$. As $\kappa$ increases, the soliton becomes shorter in time. At some value of $\kappa$ one has to switch from analytic representation to the numerical solution of Eq. (20). The soliton as a localized formation can be traced up to the largest finite $\kappa$ at which it vanishes mainly because of the resonant radiation. For idealized dispersion profiles, e.g., for Drude's dispersion, the resonant radiation is absent. In that case one observes a tendency to cusp formation, as predicted by the exact solutions known from $[14,19,20]$.

Before proceeding further, we note that usually the corrections to the solitary wave solutions of Eq. (20) introduced by the nonlinear dispersion and the backward-propagating waves are small. Analyzing Eq. (21), we can see that the $\eta$ term is favored by a steep group-velocity dispersion and short pulse durations. We thus consider ultrashort pulses with the carrier frequency well within the anomalous dispersion region. An illustrative example of a single-cycle (full width at half maximum) solitary wave solution is shown in Fig. 3(a). It was calculated for the bulk fused silica dispersion at the carrier wavelength $\lambda=2.5 \mu \mathrm{m}$. The nonlinear coefficient $n_{2}=2.7 \times 10^{-16} \mathrm{~cm}^{2} / \mathrm{W}$. For comparison, the corresponding fundamental soliton solution given by Eq. (23) with $t_{0}=5.1 \mathrm{fs}$ and $\sqrt{n_{2}} f(0)=0.063$, peak power $\approx 1.46 \times 10^{13} \mathrm{~W} / \mathrm{cm}^{2}$, is shown in Fig. 3(a) by the gray curve. Surprisingly, the fundamental soliton of the integrable NLSE remains to be a reasonably good approximation to the exact solution for practically all pulse durations. The difference, however, is clearly seen in the spectral domain [Fig. 3(b)]. The relative role of the nonlinear dispersion and the backward waves as well as the numerical approach to the solitary solutions are described in the next section. 

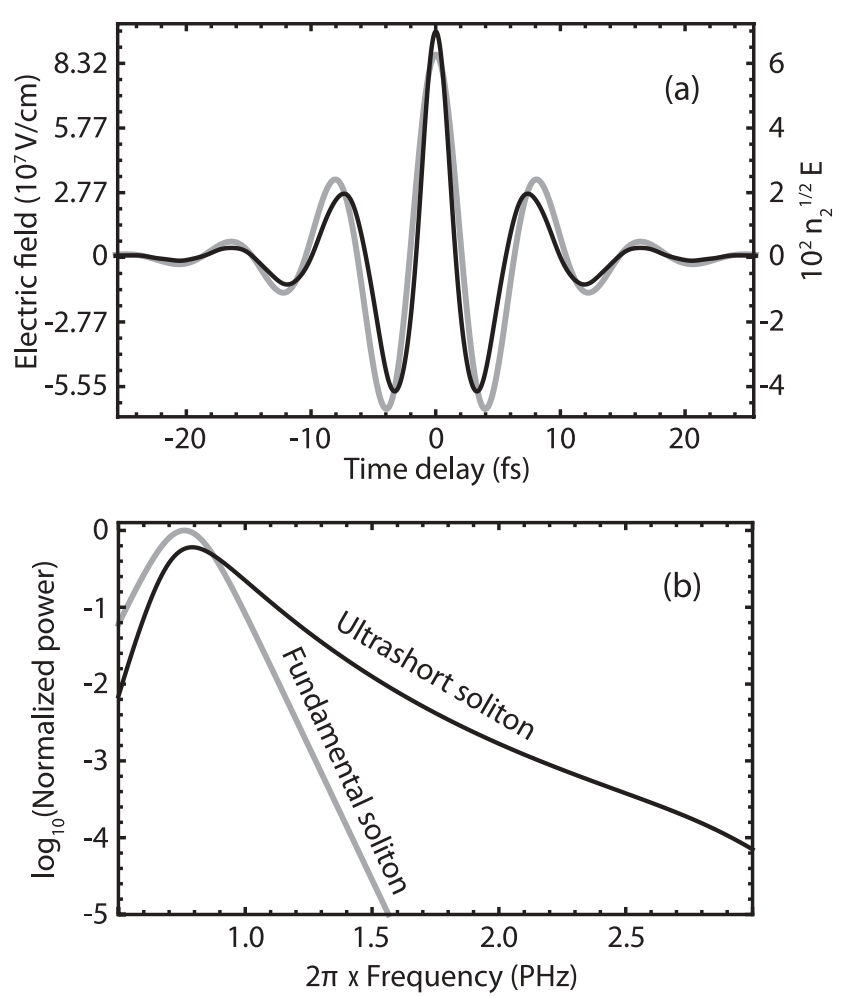

FIG. 3. (a) A single-cycle soliton at $\lambda=2.5 \mu \mathrm{m}$ is shown by the black curve. For comparison, the corresponding fundamental NLSE soliton solution is shown by the gray curve. (b) Spectral densities of the same solutions. Dispersion used here is for the bulk fused silica. Pulse parameters are given in the text.

\section{NUMERICAL SOLUTIONS}

Equation (20) is now written in a form which suggests an iterative solution:

$$
f(\tau)=\hat{\mathcal{N}}[f(\tau)], \quad(\hat{\mathcal{N}}[f])_{\Omega}=K(\Omega) n_{2}\left(|f|^{2} f\right)_{\Omega},
$$

where

$$
K(\Omega)=\frac{n\left(\omega_{0}\right)}{n\left(\omega_{0}+\Omega\right)+\eta(\Omega)} \frac{\omega_{0}+\Omega}{[\kappa-\tilde{\beta}(\Omega)] c},
$$

and we recall that $\kappa-\tilde{\beta}(\Omega)$ is positively defined in the absence of resonant frequencies.

Iterations start with the fundamental soliton solution (23). The simplest iterative scheme, $f_{n+1}=\hat{\mathcal{N}}\left[f_{n}\right]$, unfortunately diverges. In order to ensure the convergence, one can apply the so-called spectral renormalization method developed for the NLSE [32]. In the course of iterations, the transition from $f_{n}$ to $f_{n+1}$ is performed in two steps:

$$
f_{n+1 / 2}=\hat{\mathcal{N}}\left[f_{n}\right], \quad f_{n+1}=s_{n} f_{n+1 / 2},
$$

where the rescaling factor $s_{n}$ in the second step is chosen in such a way that $\left\langle f_{n+1} \mid f_{n+1}\right\rangle=\left\langle f_{n} \mid f_{n}\right\rangle$, for a suitably defined scalar product, e.g., the time-averaged value of $|f|^{2}$. A better choice is given by the scalar product naturally related to Eq. (15). After rescaling, all $f_{n}(\tau)$ belong to a corresponding "unit sphere." This option considerably improves the conver-

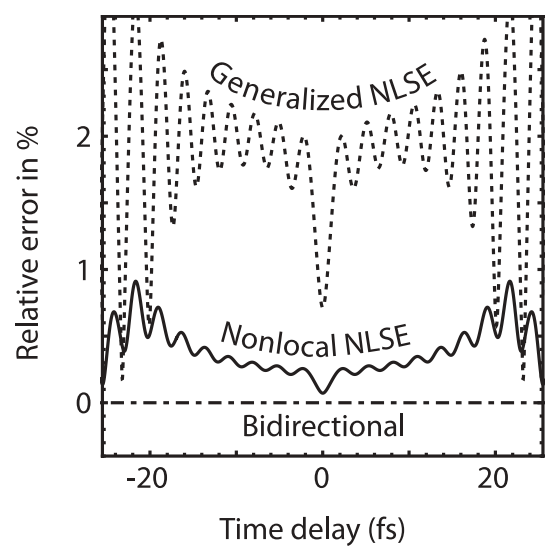

FIG. 4. The relative error across the soliton. Soliton profiles obtained using different versions of the generalized NLSE slightly deviate from the profile given by the bidirectional equation. The "zero-error" dash-dotted line corresponds to the exact soliton of Eq. (20), presented in Fig. 3 by the black curve. The solid line results from using the nonlocal Eq. (25), while the dotted line results from using the local Eq. (24). The inaccuracy of the classical NLSE (22) (not shown here) is considerably higher.

gence. If the iterations converge to $f_{\infty}$ and $s_{\infty}$, we have

$$
f_{\infty}=s_{\infty} \hat{\mathcal{N}}\left[f_{\infty}\right] \Rightarrow \sqrt{s_{\infty}} f_{\infty}=\hat{\mathcal{N}}\left[\sqrt{s_{\infty}} f_{\infty}\right]
$$

just because $\hat{\mathcal{N}}[f]$ represents a cubic nonlinearity. It follows that

$$
f(\tau)=\sqrt{s_{\infty}} f_{\infty}(\tau)
$$

solves the equation $f(\tau)=\hat{\mathcal{N}}[f(\tau)]$, which then takes the soliton shape. Finding a solution, such as the one presented in Fig. 3, typically requires several hundreds of iterations.

Figure 4 shows the relative error versus the retarded time for the different solutions considered here. The error is defined as $\|F(\tau) / f(\tau)-1\|$, where one of the simplified equations (22), (24), or (25) yields $F(\tau)$, whereas $f(\tau)$ solves the full Eq. (20). The norm $\|F\|=\sqrt{\langle F \mid F\rangle}$ is identical to that used for rescaling the successive iterations. We see that the contribution of both the nonlinear dispersion and the backward propagating waves remains relatively small even for a single-cycle pulse.

\section{CONCLUSIONS}

In conclusion, we analyzed the propagation of few-cycle optical solitons in nonlinear media with an anomalous, but otherwise arbitrary, dispersion and a cubic nonlinearity. The analytic signal representation yields a natural way to ignore generation of the third harmonic for a smooth, nonresonant dispersion profile. In this approach, the nonlinear wave equation for optical pulses in fibers is transformed to a simplified propagation equation without introducing a carrier frequency. Neither the slowly varying envelope approximation nor the unidirectional approximation are used.

The resulting Eq. (15) is a bidirectional nonlinear wave equation. This equation applies directly to the electric field and describes pulses with arbitrary durations, as long as the third harmonic generation can be neglected. Solitary wave solutions of Eq. (15) have been studied numerically using the spectral renormalization method. Each solution can be 
effectively traced up to a few-cycle duration. We compared them with solutions given by the envelope equations.

Our approach allowed us to consider separately contributions of linear and effective nonlinear dispersion, the selfsteepening term, and the term resulting from the backward wave propagation. This analysis has revealed that the main contribution comes from the higher-order linear dispersion, and, to a lesser extent, from the self-steepening term. The influence of the nonlinear dispersion and the backward waves can also be noticed for ultrashort few-cycle pulses. The reason the ultrashort solitons are finally destroyed is Cherenkov radiation.

\section{ACKNOWLEDGMENTS}

Sh.A. gratefully acknowledges support by the DFG Research Center MATHEON under project D 14. N.A. acknowledges the support of the Australian Research Council (Discovery Project DP110102068) and the Volkswagen Stiftung. He is also a recipient of the Alexander von Humboldt Award.

\section{APPENDIX: ENVELOPE EQUATIONS}

In this Appendix we list the commonly used envelope equations and explain in more detail how the envelope solitons are related to those obtained from Eq. (20). The pulse envelope $\psi(z, \tau)$ is defined by the standard expression

$$
E(z, t)=\frac{1}{2} \psi\left(z, t-\beta_{1} z\right) e^{i\left(\beta_{0} z-\omega_{0} t\right)}+\text { c.c. }
$$

In contrast to our ansatz (18), the envelope is subject to an additional approximation of one-way propagation and therefore can be found from the first-order equation rather than from the bidirectional Eq. (15). The solitary solutions of the generalized NLSEs are derived assuming

$$
\psi(z, \tau)=f(\tau) e^{i \kappa z}
$$

Let us now consider particular cases.

\section{Classical NLSE}

By inserting Eq. (A2) into the standard NLSE,

$$
i \partial_{z} \psi+\frac{\beta_{2}}{2}\left(i \partial_{\tau}\right)^{2} \psi+\frac{\omega_{0}}{c} n_{2}|\psi|^{2} \psi=0,
$$

after the transformation into the frequency domain we obtain Eq. (22). Comparing this to Eq. (20), we can see the two differences. First, the deviation $\tilde{\beta}(\Omega)$ is replaced by its leading term (SVEA)

$$
\Omega \ll \omega_{0}, \quad \tilde{\beta}(\Omega) \approx \frac{\beta_{2}}{2} \Omega^{2} .
$$

Second, the dispersion in the right-hand side of Eq. (20) is completely ignored.

\section{Generalized NLSE}

Following Ref. [39] we can write the generalized NLSE in the form

$$
i \partial_{z} \psi+\hat{\mathcal{D}} \psi+\frac{\omega_{0}+i \partial_{\tau}}{c} n_{2}|\psi|^{2} \psi=0,
$$

where

$$
\hat{\mathcal{D}}=\sum_{m=2}^{\infty} \frac{\beta_{m}}{m !}\left(i \partial_{\tau}\right)^{m}
$$

denotes the complete dispersion operator and all dissipative terms are removed to allow for solitary solutions. Using Eq. (A2) after the transformation into the frequency domain we obtain Eq. (24), which fully accounts for the higherorder linear dispersion. However the nonlinear dispersion is only taken into account by the self-steepening term. Interestingly, the NLSE equipped with the self-steepening term

$$
i \partial_{z} \psi+\frac{\beta_{2}}{2}\left(i \partial_{\tau}\right)^{2} \psi+\frac{\omega_{0}+i \partial_{\tau}}{c} n_{2}|\psi|^{2} \psi=0
$$

is completely integrable [44]. The generalized fundamental soliton of this equation is given by the relation (A2), where similarly to Eq. (23) we can write

$$
\sqrt{n_{2}} f(\tau)=\sqrt{\frac{2 \kappa c}{\omega_{0}}} A\left(\frac{\tau}{t_{0}}\right), \quad t_{0}(\kappa)=\sqrt{\frac{\left|\beta_{2}\right|}{2 \kappa},}
$$

except for the amplitude function being replaced by

$$
\left|A\left(\tau / t_{0}\right)\right|=\sqrt{\frac{2 \omega_{0} t_{0}}{\omega_{0} t_{0}+\cosh \left(2 \tau / t_{0}\right) \sqrt{\left(\omega_{0} t_{0}\right)^{2}+1}}},
$$

and

$$
\arg A\left(\tau / t_{0}\right)=-3 \arctan \left[\frac{\tanh \left(\tau / t_{0}\right)}{\omega_{0} t_{0}+\sqrt{\left(\omega_{0} t_{0}\right)^{2}+1}}\right] .
$$

The fundamental soliton solution is recovered from here in the SVEA limit, $\omega_{0} t_{0} \gg 1$.

\section{Nonlocal NLSE}

The nonlocal generalization of the NLSE [38,40-43] can be written as

$$
i \partial_{z} \psi+\hat{\mathcal{D}} \psi+\frac{n\left(\omega_{0}\right)}{c} \frac{\omega_{0}+i \partial_{\tau}}{n\left(\omega_{0}+i \partial_{\tau}\right)} n_{2}|\psi|^{2} \psi=0,
$$

where the pseudodifferential operator in the nonlinear term can be represented the same way as the dispersion operator

$$
\frac{\omega_{0}+i \partial_{\tau}}{n\left(\omega_{0}+i \partial_{\tau}\right)}=\sum_{m=0}^{\infty} \frac{\gamma_{m}}{m !}\left(i \partial_{\tau}\right)^{m} .
$$

This expression is valid provided that the series converges, i.e., provided that pulse width in the frequency domain is smaller than the convergence radius. The latter is determined by the "hidden" singularities of $1 / n(\omega)$ in the complex plane. When the pulse spectral width is too large, both $n\left(\omega_{0}+i \partial_{\tau}\right)$ and $\hat{\mathcal{D}}$ are nonlocal operators. These pseudodifferential operators should be understood as convolutions. In any case, by using Eq. (A2) for the localized solutions and transforming to the frequency domain, we obtain Eq. (25) in which only the $\eta$ term is missing as compared to the full bidirectional Eq. (20). 
[1] A. Hasegawa, Optical Solitons in Fibers (Springer, New York, 1980).

[2] N. Akhmediev and A. Ankiewicz, Solitons: Nonlinear Pulses and Beams (Chapman and Hall, London, 1997).

[3] V. E. Zakharov and A. B. Shabat, Sov. Phys. JETP 34, 62 (1972).

[4] T. Brabec and F. Krausz, Rev. Mod. Phys. 72, 545 (2000).

[5] K. Akimoto, J. Phys. Soc. Jpn. 65, 2020 (1996).

[6] M. Hentschel, R. Kienberger, C. Spielmann, G. A. Reider, N. Milosevic, T. Brabec, P. Corkum, U. Heinzmann, M. Drescher, and F. Krausz, Nature (London) 414, 509 (2001).

[7] P. M. Paul, E. S. Toma, P. Breger, G. Mullot, F. Augé, P. Balcou, H. G. Muller, and P. Agostini, Science 292, 1689 (2001).

[8] M. Drescher, M. Hentschel, R. Kienberger, G. Tempea, C. Spielmann, G. A. Reider, P. B. Corkum, and F. Krausz, Science 291, 1923 (2001).

[9] V. N. Serkin and A. Hasegawa, Phys. Rev. Lett. 85, 4502 (2000).

[10] E. M. Gromov and V. I. Talanov, Chaos 10, 551 (2000).

[11] M. Gedalin, T. C. Scott, and Y. B. Band, Phys. Rev. Lett. 78, 448 (1997).

[12] C. E. Zaspel, Phys. Rev. Lett. 82, 723 (1999).

[13] K. Porsezian and K. Nakkeeran, Phys. Rev. Lett. 76, 3955 (1996).

[14] S. Amiranashvili, U. Bandelow, and N. Akhmediev, Phys. Rev. A 84, 043834 (2011).

[15] H. Leblond and F. Sanchez, Phys. Rev. A 67, 013804 (2003).

[16] E. V. Kazantseva, A. I. Maimistov, and J.-G. Caputo, Phys. Rev. E 71, 056622 (2005).

[17] A. Sakovich and S. Sakovich, J. Phys. A 39, L361 (2006).

[18] H. Leblond and D. Mihalache, Phys. Rev. A 79, 063835 (2009).

[19] S. A. Skobelev, D. V. Kartashov, and A. V. Kim, Phys. Rev. Lett. 99, 203902 (2007).

[20] S. Amiranashvili, A. G. Vladimirov, and U. Bandelow, Phys. Rev. A 77, 063821 (2008).

[21] H. Leblond, H. Triki, and D. Mihalache, Phys. Rev. A 84, 023833 (2011).

[22] A. E. Kaplan and P. L. Shkolnikov, Phys. Rev. Lett. 75, 2316 (1995).
[23] A. I. Maimistov, Quantum Electron. 30, 287 (2000).

[24] H. Leblond, S. V. Sazonov, I. V. Mel'nikov, D. Mihalache, and F. Sanchez, Phys. Rev. A 74, 063815 (2006).

[25] S. A. Kozlov and S. V. Sazonov, JETP 84, 221 (1997).

[26] T. Schäfer and C. E. Wayne, Physica D 196, 90 (2004).

[27] N. Akhmediev and M. Karlsson, Phys. Rev. A 51, 2602 (1995).

[28] D. V. Skryabin and A. V. Gorbach, Rev. Mod. Phys. 82, 1287 (2010).

[29] P. M. Goorjian and A. Taflove, Opt. Lett. 17, 180 (1992).

[30] P. M. Goorjian, A. Taflove, R. M. Joseph, and S. C. Hagness, IEEE J. Quantum Electron. 28, 2416 (1992).

[31] J. C. A. Tyrrell, P. Kinsler, and G. H. C. New, J. Mod. Opt. 52, 973 (2005).

[32] M. J. Ablowitz and T. P. Horikis, Eur. Phys. J. Special Topics 173, 147 (2009).

[33] V. E. Zakharov and E. A. Kuznetsov, JETP 86, 1035 (1998).

[34] D. E. Vakman and L. A. Vainshtein, Usp. Fiz. Nauk 20, 1002 (1977)

[35] S. Amiranashvili and A. Demircan, Phys. Rev. A 82, 013812 (2010).

[36] V. E. Zakharov, V. S. L'vov, and G. Falkovich, Kolmogorov Spectra of Turbulence I: Wave Turbulence, Springer Seriers in Nonlinear Dynamics (Springer, Berlin, 1992).

[37] G. P. Agrawal, Nonlinear Fiber Optics, 4th ed. (Academic, New York, 2007).

[38] S. Amiranashvili, U. Bandelow, and A. Mielke, Opt. Commun. 283, 480 (2010).

[39] T. Brabec and F. Krausz, Phys. Rev. Lett. 78, 3282 (1997).

[40] M. Kolesik, J. V. Moloney, and M. Mlejnek, Phys. Rev. Lett. 89, 283902 (2002).

[41] N. Karasawa, S. Nakamura, N. Nakagawa, M. Shibata, R. Morita, H. Shigekawa, and M. Yamashita, IEEE J. Quantum Electron. 37, 398 (2001).

[42] P. Kinsler and G. H. C. New, Phys. Rev. A 67, 023813 (2003).

[43] M. Kolesik and J. V. Moloney, Phys. Rev. E 70, 036604 (2004).

[44] H.-Q. Zhang, B.-G. Zhai, and X.-L. Wang, Phys. Scr. 85, 015007 (2012). 